\title{
SEPARATION PROPERTIES OF CONVEX CONES ${ }^{1}$
}

V. L. KLEE, JR.

1. Introduction. If $A$ and $B$ are convex subsets of a topological linear space $E$, we say that $A$ and $B$ can be separated by a hyperplane provided $E$ admits a continuous linear functional $f$, not identically zero, such that $\sup _{A} f \leqq \inf _{B} f$. Except in rather special cases, the known separation theorems require that $A$ and $B$ have no common point, and thus fail to cover the interesting case in which $A$ and $B$ are closed convex cones whose intersection and common vertex is the origin $\phi$. This case is discussed in the present note. Our main results, extending theorems of Aronszajn [1] and Tagamlitzki [6], are proved in $\S 2 ; \S 3$ contains examples showing that these results cannot be substantially improved.

In addition to standard notation and terminology, we employ the following: A $\phi$-cone in a topolugical linear space is a closed convex cone having vertex $\phi$; for a $\phi$-cone $A, A^{\prime}$ will denote the linear subspace $A \cap-A$. Set-theoretic sum and difference are indicated by $\cup$ and $\backslash$ respectively, + and - being reserved for the linear operations. For points $x$ and $y$ of a linear space, $[x, y] \equiv\{t x+(1-t) y: 0 \leqq t \leqq 1\}$, ]$x, y[\equiv\{t x+(1-t) y: 0<t<1\}$, etc.

2. The separation theorems. Our main results, (2.5) and (2.7), will be based on several propositions about $\phi$-cones. The first of these can be proved by modification of an argument in [5, p. 452] (see also $[4$, p. 78]):

(2.1) If $A$ and $B$ are $\phi$-cones in a topological linear space, $A$ is locally compact, and $A \cap B=\{\phi\}$, then $A-B$ is closed.

(2.2) If $A$ is a $\phi$-cone in a locally convex topological linear space $E$ and $Q$ is a nonempty compact convex subset of $A$ which misses $A^{\prime}$, then $E$ admits a continuous linear functional $f$ such that $f \geqq 0$ on $A$ and $f>0$ on $Q$.

PRoof. Let $V$ be the family of all convex open sets $U \ni \phi$ such that $Q+U$ misses $A^{\prime}$, and for each $U \in V$ let $A_{U}$ be the convex hull of $\left(A \backslash A^{\prime}\right) \cup(Q+U)$. An elementary argument (similar to that of [5, p. 459]) shows that for some $V \in \mathcal{V}, \phi \notin A_{V}$. Since the interior of $A_{V}$ is nonempty, it then follows from the basic separation theorem

Presented to the Society, December 30, 1954 ; received by the editors July 3, 1954.

1 Sponsored by the Office of Ordnance Research, U. S. Army, under Contract DA-04-200-ORD-292. 
( $[4$, p. 69$]$ and $[5$, p. 456$])$ that $E$ admits a continuous linear functional $f$, not identically zero, such that $f \geqq 0$ on $A_{V}$. It is easy to verify that $f \geqq 0$ on $A$ and $f>0$ on $Q$.

(2.3) If $A$ is a locally compact $\phi$-cone in a locally convex topological linear space $E$, then there are locally compact $\phi$-cones $A_{1}$ and $A_{2}$ in $E$ such that $A_{1}^{\prime}=A_{1}, A_{1}^{\prime}=\{\phi\}=A_{1} \cap A_{2}$, and $A_{1}+A_{2}=A$.

Proof. $A^{\prime}$ is a locally compact linear subspace of $E$, hence is finitedimensional, and thus admits a complementary closed linear subspace $L$ in $E$. With $A_{1}=A^{\prime}$ and $A_{2}=A \cap L$, it can be verified that the sets $A_{i}$ have the stated properties.

The proof of the next proposition is due to J. Dieudonné and the author, independently. Dieudonné also observed that it could be used to prove the theorem (2.5).

(2.4) If $A_{2}$ is a locally compact $\phi$-cone in a locally convex topological linear space $E$, and $A_{2}^{\prime}=\{\phi\}$; then there is a.compact convex set $J \subset E \backslash\{\phi\}$ such that $A_{2}=[0, \infty[J$.

ProOF. By local convexity of $E$ and local compactness of $A_{2}$, there is a convex subset $K$ of $E$ such that $\phi$ is interior to $K$ and $A_{2} \cap K$ is compact. Let $F$ be the intersection of $A_{2}$ with the boundary of $K$ and let $J$ be the closed convex hull of $F$. Then clearly $A_{2}=[0, \infty[J$, and compactness of $J$ follows from a result of Bourbaki [4, p. 80]. Now if $\phi \in J$, then $\phi$ is an extreme point of $J$, for $J \subset A$ and $A^{\prime}=\{\phi\}$. Then from a theorem of Milman [4, p. 84] it follows that $\phi \in F$, a contradiction completing the proof.

We remark that (2.3) and (2.4) have valid converses which do not require local convexity of $E$. [6].

A very special case of the theorem below was given by Tagamlitzki

(2.5) Theorem. Suppose $A$ and $B$ are $\phi$-cones in a locally convex topological linear space $E, A$ is locally compact, and $A \cap B=\{\phi\}$. Then $E$ admits a continuous linear functional $f$ such that $f<0$ on $A \backslash A^{\prime}, f=0$ on $A^{\prime} \cup B^{\prime}$, and $f \geqq 0$ on $B \backslash B^{\prime}$.

Proof. By (2.1), $B \backslash A$ is closed, and hence is a $\phi$-cone. Let $A_{1}$ and $A_{2}$ be as in (2.3) and let $J$ be as in (2.4). Then $(B-A)^{\prime}=B^{\prime}-A^{\prime}$ $=B^{\prime}-A_{1}$, so that if $-J$ intersects $(B-A)^{\prime}$ it must be true that $B^{\prime}$ intersects $J+A_{1}$. Since $A \cap B=\{\phi\}$ and $J+A_{1} \subset A_{2}$, this is impossible, and it follows that $-J$ misses $(B-A)^{\prime}$. Thus, by (2.2), $E$ admits a continuous linear functional $f$ such that $f \geqq 0$ on $B-A$ and $f>0$ on $-J$. It is easy to verify that $f$ has the desired properties.

Now if, in (2.5), $B$ is also locally compact, then $E$ admits a continuous linear functional $g$ such that $g<0$ on $B \backslash B^{\prime}, g=0$ on $B^{\prime} \cup A^{\prime}$, 
and $g \geqq 0$ on $A \backslash A^{\prime} ; f-g$ is then a linear functional $<0$ on $A \backslash A^{\prime},=0$ on $A^{\prime} \cup B^{\prime}$, and $>0$ on $B \backslash B^{\prime}$. This stronger type of separation is also available in another setting, which we now discuss. The lemma needed is

(2.6) Suppose $E$ is a complete metric linear space, $C$ is a closed convex subset of $E, \phi \in C$, and $x_{\alpha}$ is a sequence of points of $C$. Then there are a point $p$ of $C$ and a sequence $t_{\alpha}$ of positive numbers such that for each $i,\left[p-t_{i} x_{i}, p+t_{i} x_{i}\right] \subset C$. If $F$ is a set of linear functionals on $E$ such that every member of $F$ which is constant on $\left\{x_{1}, x_{2}, \ldots\right\}$ is constant on $C$, then $f p \in] \inf _{C} f, \sup _{C} f[$ for each $f \in F$.

Proof. Let $\rho$ be a complete invariant metric on $E$. For each $i$, choose $t_{i}$ such that $0<t_{i}<2^{-i-1}>\rho\left(\phi, t_{i} x_{i}\right)$. Then the series $\sum_{1}^{\infty} t_{i} x_{i}$ converges to a point $p \in C$, and for each $i$ it is true that $\left[p-t_{i} x_{i}\right.$, $\left.p+t_{i} x_{i}\right] \subset C$. Now consider an arbitrary $f \in F$. If $f$ is constant on $\left\{x_{1}, x_{2}, \cdots\right\}$, then of course $\left.f p \in\right] \inf _{c} f, \sup _{c} f[$. If for some $i$, $f x_{i}>0$, then $\inf _{C} f \leqq f\left(p-x_{i}\right)<f p<f\left(p+x_{i}\right) \leqq \sup _{C} f$. A similar statement holds if $f x_{i}<0$ for some $i$, so the proof is complete.

(2.7) Theorem. Suppose $E$ is a separable normed linear space, $A$ and $B$ are $\phi$-cones in $E, A$ is locally compact, and $A \cap B=\{\phi\}$. Then $E$ admits a continuous linear functional $f$ such that $f<0$ on $A \backslash A^{\prime}$, $f=0$ on $A^{\prime} \cup B^{\prime}$, and $f>0$ in $B \backslash B^{\prime}$.

Proof. Let $D=B-A$. By (2.1), $D$ is a $\phi$-cone. Let $C$ be the set of all $g \in E^{*}$ such that $g \geqq 0$ on $D$. $C$ is a closed convex subset of $E^{*}$, and by [2, p. 124], $C$ is separable in the $w^{*}$-topology. Hence by $(2.6)$ there is an $f \in C$ such that $f x \in] \inf _{o \in C} g x$, sup $\operatorname{suc}_{o \in C} g x$ [ for each $x \in E$. For $x \in D \backslash D^{\prime}$ we have inf $g_{a \in C} g x \geqq 0$ and (by (2.2)) sup $_{o \in C} g x>0$, whence $f x>0$. Thus $f$ is a continuous linear functional on $E$ such that $f \geqq 0$ on $D$ and $f>0$ on $D \backslash D^{\prime}$. Since $D^{\prime}=(B-A)^{\prime}=B^{\prime}-A^{\prime}$, it is easy to verify that $f$ has the desired properties.

If $L$ is a linear space and $p$ is a positively homogeneous subadditive functional on $L$, then, by the Hahn-Banach theorem, $L$ admits a linear functional $f \leqq p$. Aronszajn [1] showed that if $p x+p(-x)$ $=n x>0$ for each $x \in L \backslash\{\phi\}$, and $L$ is separable under the norm $n$, then $L$ admits a linear functional $f$ such that $f x<p x$ whenever $x \in L \backslash\{\phi\}$. Bonsall [3] showed by an example that the assumption of separability cannot be discarded. Aronszajn's result follows easily from (2.7), by application of (2.7) to the space $L \times R$. (For the type of argument needed, see $[5$, p. 462$]$.)

3. Remarks and examples. Let $E$ be either the hyper-Hilbert space $l^{2} \aleph_{1}$ or the complete separable locally convex metric linear space $(s)$ of 
all sequences of real numbers [2, p. 10], and let $B$ be the set of all non-negative members of $E$. Then $B$ is a $\phi$-cone and $B^{\prime}=\{\phi\}$, but $E$ does not admit any linear functional $f$ such that $f>0$ on $B \backslash\{\phi\}$. This shows that $E$ must be taken normed and separable in (2.7), and helps to clarify the role played by local compactness in (2.5).

The separation theorems (2.5) and (2.7) assert more than the possibility of separating $A$ and $B$ by a hyperplane, for in each case we have not merely $f \leqq 0$ on $B$ but also $f<0$ on $B \backslash B^{\prime}$. We shall now develop an example which shows that without local compactness of at least one of the $\phi$-cones involved, even the weakest sort of separation may not be possible.

A subset of a linear space is said to be linearly bounded provided its intersection with every line is contained in some bounded subset of the line. For later use, we mention the following fact, whose proof is left to the reader:

(3.1) If $E$ is a topological linear space, $M$ is a hyperplane in $E \backslash\{\phi\}$, and $J$ is a convex subset of $M$, then $[0, \infty[J$ is closed if and only if $J$ is closed and linearly bounded.

Now if $E$ is an arbitrary nonreflexive separable Banach space, then $E$ contains a disjoint pair of bounded closed convex sets which cannot be separated by any hyperplane [5, p. 881]; application of (3.1) produces in $E$ a pair of $\phi$-cones $A$ and $B$ which cannot be separated by any hyperplane, even though $A \cap B=\{\phi\}$. In reflexive spaces, on the other hand, disjoint bounded closed convex sets can always be separated by a hyperplane. Nevertheless, we have

(3.2) Hilbert space $H$ contains a disjoint pair of linearly bounded closed convex sets which cannot be separated by any hyperplane.

Proof. We may regard $H$ as $H_{1} \times H_{2}$, where each $H_{i}$ is a sequential Hilbert space. Let $Q$ be a compact convex subset of $H_{2}$ which contains the origin $\phi_{2}$, but cannot be supported by a hyperplane at $\phi_{2}$. Let $p$ be a point of $H_{2}$ such that ]0, $\infty$ [p misses $-Q$. Let the points $\delta_{i}$ of $H_{1}$ be given by $\delta_{i}{ }^{i}=1$ and $\delta_{i}{ }^{i}=0$ for $i \neq j$, and let $a_{\alpha}$ and $b_{\alpha}$ be sequencies of positive numbers, with $b_{\alpha} \rightarrow 0$. (A further restriction on $a_{\alpha}$ will be added later.) Let $C=\left\{\left(x, \phi_{1}\right): x \in H_{1}\right.$ and $x^{i} \in\left[0, a_{i}\right]$ for each $i\}$ and let $D$ be the closed convex hull of $\left\{\left(a_{n} \delta_{n}, q+b_{n} p\right): n\right.$ a positive integer, $q \in Q\}$. It can be verified that $C$ and $D$ are linearly bounded closed convex subsets of $H=H_{1} \times H_{2}$.

Now suppose $f$ is a continuous linear functional on $H$ such that $\sup _{C} f \leqq \inf _{D} f$. Then for each $n, f\left(a_{n} \delta_{n}, \phi_{2}\right) \leqq f\left(a_{n} \delta_{n}, q+b_{n} p\right)$ for all $q \in Q$, whence $f\left(\phi_{1}, q\right) \geqq 0$ for all $g \in Q$. Since $\phi_{2}$ is a nonsupport point of $Q$ in $H_{2}$, it follows that $f\left(\phi_{1}, y\right)=0$ for all $y \in H_{2}$. But then $f\left(a_{i} \delta_{i}, \phi_{2}\right)$ $\leqq f\left(a_{j} \delta_{j}, \phi_{2}\right)$ for all $i$ and $j$, and it follows that $f$ must be identically 
zero. We still must show that, with appropriate specification of $a_{\alpha}$, $C$ misses $D$.

Consider an arbitrary point $\left(x, \phi_{2}\right)$ of $C$. If this is also a point of $D$, there must be positive numbers $t_{j}{ }^{i}(1 \leqq i, j<\infty)$ and points $q_{j}$ of $Q$ such that $u_{\alpha} \rightarrow x$ and $v_{\alpha} \rightarrow \phi_{2}$, where for each $j$ it is true that $\sum_{i=1}^{\infty} t_{j}^{i}=1, \quad u_{j}=\sum_{i=1}^{\infty} t_{j}{ }^{i} a_{i} \delta_{i}$, and $v_{j}=\left(\sum_{i=1}^{\infty} t_{j}{ }^{i} b_{i}\right) p+q_{j}$. Since $v_{\alpha} \rightarrow \phi_{2}, Q$ is compact, and $] 0, \infty\left[p+Q\right.$ misses $\phi_{2}$, it is necessary that $\lim _{j \rightarrow \infty} \sum_{i=1}^{\infty} t_{j}{ }^{i} b_{i}=0$. Since each $b_{i}$ is positive, that implies $\lim _{j \rightarrow \infty} t_{j}{ }^{i}$ $=0$ for each $i$; and with $u_{\alpha} \rightarrow x$, this implies $x=\phi_{1}$. Thus with no further restriction on the sequences $a_{\alpha}$ and $b_{\alpha}$, it must be true that $C \cap D \subset\left\{\left(\phi_{1}, \phi_{2}\right)\right\}$. Now the square of the distance from $u_{j}$ to $\phi_{1}$ is $\sum_{i=1}^{\infty}\left(t_{j}{ }^{i} a_{i}\right)^{2}$, and for this to be less than 1 it must be true that $t_{j}^{i}<a_{i}^{-1}$ for all $i$. Since $\sum_{i=1}^{\infty} t_{j}^{i}=1$ for each $i$, then if $a_{\alpha}$ is any sequence of positive numbers such that $\sum_{1}^{\infty} a_{i}^{-1}<1$, the resultant sets $C$ and $D$ have no points in common. This completes the proof.

From (3.1) and (3.2) we now have

(3.3) Hilbert space contains a pair of $\phi$-cones $A$ and $B$ which cannot be separated by a hyperplane, even though $A^{\prime}=B^{\prime}=A \cap B=\{\phi\}$.

A slightly more careful argument would have shown that in (3.3), $A$ and $B$ can be so constructed that $x \cdot y>0$ whenever

$$
\{x, y\} \subset A \cup B \backslash\{\phi\} .
$$

In closing, a word about the role played in separation theorems by the assumption that the sets are disjoint. The basic separation theorem $[4$, p. $69 ; 5$, p. 456] asserts that if $C$ and $D$ are convex and the interior of $C$ is nonempty and misses $D$, then $C$ and $D$ can be separated by a hyperplane. Here $C \cap D$ need not be empty. But compactness will not suffice for theorems of this type, as is seen from easily constructed examples of compact convex sets $C$ and $D$ in Hilbert space which cannot be separated by a hyperplane, even though, in the first case, $D$ is merely an extreme point of $C$, and, in the second case, $D$ is a line segment such that $C \cap D$ is a point of $C$ at which $C$ can be supported by a hyperplane. Relevance of these examples to the case of $\phi$-cones $A$ and $B$, and the condition that $A \cap B=\{\phi\}$, can be seen in terms of (3.1). Only in finite-dimensional spaces is the situation especially simple, as described in

(3.4) Suppose $E$ is a finite-dimensional topological linear space and $A_{1}$ and $A_{2}$ are convex subsets of $E$ whose union is not contained in any hyperplane. For each $i$, let $A_{i}{ }^{0}$ be the interior of $A_{i}$, relative to the smallest linear manifold containing it. Then $A_{1}$ and $A_{2}$ can be separated by $a$ hyperplane if and only if $A_{1}{ }^{0}$ misses $A_{2}{ }^{0}$. 


\section{REFERENCES}

1. N. Aronszajn, Sur quelques problèmes concernant les espaces de Minkowski et les espaces vectoriels généraux, Atti della Accademia Nazionale dei Lincei. Rendiconti (6) vol. 26 (1937) pp. 374-376.

2. Stefan Banach, Theorie des operations lineaires, Warsaw, 1932.

3. F. F. Bonsall, $A$ note on subadditive functionals, J. London Math. Soc. vol. 29 (1954) pp. 125-126.

4. N. Bourbaki, Espaces vectoriels topologiques, Actualités Scientifiques et Industrielles, no. 1189, Paris, 1953.

5. V. L. Klee, Jr., Convex sets in linear spaces, Duke Math. J. vol. 18 (1951) pp. 443-466, 875-883.

6. Y. Tagamlitzki, Übertragung des Minkowskischen Stiutzebenensatzes auf Hilbertsche Räume, C. R. Acad. Bulgare Sci. vol. 4 (1951) no. 2-3 (1953) pp. 5-8.

UNIVERSITY OF WASHINGTON

\section{A NOTE ON HAAR MEASURE}

\section{HARRY F. DAVIS}

We prove a direct sum theorem for Haar measure which is a generalization, to arbitrary locally compact topological groups, of a theorem for Lie groups due to Mostow [3]. It is well known that the Haar integral on a compact group may be obtained by a simple averaging process. On a vector group, the Haar integral is essentially the Lebesgue integral. On a discrete group it is summation. Our theorem asserts that the formation of a Haar integral on any locally compact group must be a composite of these three processes. Hence no locally compact group exists for which the Haar integral must be constructed in an essentially more novel manner.

THEOREM. Given any locally compact topological group $G$, there exists a compact subgroup $K$, a subspace $E$ which is homeomorphic to an ndimensional Euclidean space, and a discrete subset $D$, such that the mapping $\theta(a, b, c)=a b c$ is a homeomorphism of the Cartesian product $D \times E \times K$ with $G$, and carries the product measure $m_{D} \times m_{E} \times m_{K}$ over to $m_{G}$. Here $m_{D}$ is discrete measure in $D, m_{E}$ is Lebesgue measure in $E$ relative to suitable coordinates, $m_{K}$ is Haar measure in $K$, and $m_{G}$ is left Haar measure in $G$. (Note that $E$ need not be a subgroup.)

Received by the editors June 18, 1954.

1 The material in this paper constitutes part of a thesis submitted to the Massachusetts Institute of Technology and prepared under the direction of Professor Kenkichi Iwasawa. 